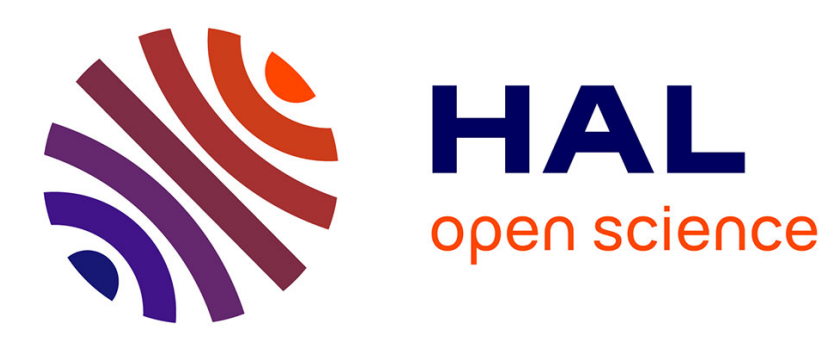

\title{
Graphene nanoribbons: photonic crystal waveguide analogy and minigap stripes
}

Henri Benisty

\section{To cite this version:}

Henri Benisty. Graphene nanoribbons: photonic crystal waveguide analogy and minigap stripes. Physical Review B: Condensed Matter and Materials Physics (1998-2015), 2009, 79 (15), pp.155409. 10.1103/PhysRevB.79.155409 . hal-00567025

\section{HAL Id: hal-00567025 \\ https://hal-iogs.archives-ouvertes.fr/hal-00567025}

Submitted on 15 Dec 2015

HAL is a multi-disciplinary open access archive for the deposit and dissemination of scientific research documents, whether they are published or not. The documents may come from teaching and research institutions in France or abroad, or from public or private research centers.
L'archive ouverte pluridisciplinaire HAL, est destinée au dépôt et à la diffusion de documents scientifiques de niveau recherche, publiés ou non, émanant des établissements d'enseignement et de recherche français ou étrangers, des laboratoires publics ou privés. 


\title{
Graphene nanoribbons: Photonic crystal waveguide analogy and minigap stripes
}

\author{
Henri Benisty \\ Laboratoire Charles Fabry de l'Institut d'Optique, CNRS, Université Paris-Sud, Campus Polytechnique, \\ RD 128, 91127 Palaiseau Cedex, France
}

(Received 20 December 2008; revised manuscript received 6 February 2009; published 3 April 2009)

\begin{abstract}
Graphene nanoribbons are one-dimensional multimode systems with quasilinear electron dispersion. We discuss why they are direct analogs of broad photonic crystal waveguides. Three kinds of dispersion regions found in band structure of the latter (Fabry-Perot region, mini-stopband region, and coupled-zone-edge regions) are traced to their equivalent in zigzag graphene nanoribbons whose edges are periodically modified. For a superperiod of $\sim 1 \mathrm{~nm}$, the zone folding arising across the nearly linear low-energy part of the electronic dispersion induces these equivalent band structures. A last analogy is the capability to induce a form of localization characterized by minigap stripes and collective flatband edges through a limited modification localized within a pair of edge rows of the nanoribbon, representing typically less than $1 \%$ of the ribbon atoms.
\end{abstract}

DOI: 10.1103/PhysRevB.79.155409

PACS number(s): 61.48.De, 62.23.Hj, 42.70.Qs, 42.82.Et

\section{INTRODUCTION: PHOTONIC CRYSTALS AND GRAPHENE NANORIBBONS}

\section{A. Common lower dimensional aspects}

Model systems of lower dimensionality are a welcome playground in condensed matter and in photonics as well, making timely comparisons ${ }^{1}$ instructive for a broader audience. One-dimensional systems exist for a long time in both domains, e.g., polydiacetylene or carbon nanotubes in the former, and optical fibers and channel waveguides in the latter. Multimode one-dimensional systems bring the general feature of interaction among their own modes. The periodicity that is either intrinsic or deterministically imprinted on such systems is a perturbation, causing the free modes to couple, as it arises in the Kronig-Peney model or quasi-freeelectron models, or in corrugated waveguides commonly used for frequency selective operation in optoelectronics, e.g., in so-called distributed feedback (DFB) laser diodes.

But graphene emerges ${ }^{2}$ as a splendid playground most notably because of the remarkable constant velocity of graphene electronic states near the Dirac points of the band structure. Furthermore, the mobility, when not limited by impurities, leads to impressive mean-free paths $\ell \sim 1 \mu \mathrm{m}$, hence across $\sim 10^{4}$ carbon atoms. The prospect of this paper, amid the blooming graphene related works, is to underline the analogies and prospects between broad photonic crystal waveguides and graphene nanoribbons (GNR). It is written from an outsider perspective (vs graphene) but we hope that the opportunity of a cross fertilization is nevertheless welcome.

The basic analogy of graphene dispersion in two dimensions (2D) with that of 2D free photons is well known. GNR are studied in great detail since the beginning of experimental graphene studies of isolated single sheet a few years ago. At first order, a GNR is thus the analog of a straight optical waveguide (well documented in textbooks ${ }^{3}$ ), and more precisely of a multimode waveguide. The energetic separation of GNR branches (modes) at the $K$ points of the Brillouin zone (BZ) (Dirac points) is on the order of $t / N_{z}$ : here $t$ is the tight-binding (TB) parameter, ${ }^{4}$ around $2.5 \mathrm{eV}$, leading formally to a total electronic bandwidth of $\pm 3 t$ (hence $6 t$ $\sim 15 \mathrm{eV}$ in graphene), and $N_{z}$ is the GNR width parameter, i.e., the number of atoms along an atomic row across the GNR.

Periodicity is nevertheless a key ingredient of GNR behavior, as identified in Nakada et al.' $\mathrm{s}^{5}$ seminal work. These authors identified the role of the GNR orientation in determining the band-structure features, and through it, the role of the edge pattern. The useful GNR period may be either canonically determined following the cut along the rows of the corresponding orientation or determined by other aspects of the system such as reconstruction, ${ }^{6}$ graphene warping, ${ }^{6}$ or externally imposed potential ${ }^{7}$ (substrate, electrodes, etc.).

Note that, to help the reader through terminology, we tabulated in Table I a set of concepts and expressions used in either of the domains or both. The text below assumes some overall knowledge of both domains but does not explicitly refer to the table, this possibility being at the reader's discretion.

If we seek the analogy with periodic waveguides, a big difference between electrons and photons is the perfect confinement offered by the crystal ions to the former particles, whereas dielectric waveguides are unable to confine all photon states. One can see laterally through a glass fiber because so many of the photon states in such a fiber are easily leaking to the outside. Furthermore, multimode fibers are mostly used as light pipes for low bandwidth uses, where coherent phenomena are undesired.

The advent of photonic crystals $(\mathrm{PhC})$, during the last two decades, revealed that properly carved dielectric patterns are able to confine light in a lossless fashion thanks to their apparent omnidirectional Bragg reflection, or more precisely thanks to their band gap, a frequency range with zero photonic density of states (DOS). Hence a photonic crystal waveguide (PhCWG) consisting of dielectric surrounded by such a photonic band-gap structure is able to confine all light states, much as electrons in solids, or also much as a confinement of photons inside a perfect metal enclosure (this latter version of confinement is unfortunately hampered, at optical frequencies, by losses of real metals; hence we do not consider it here in spite of its interest at microwave and terahertz frequencies). 
TABLE I. Concept correspondence between photonic crystal waveguides and graphene nanoribbons.

\begin{tabular}{lc}
\hline \hline Photonic crystal/optoelectronic terms & Graphene/GNR terms \\
\hline $\begin{array}{l}\text { Broad periodic waveguide guided modes, } \\
\text { dispersion branches }\end{array}$ & $\begin{array}{c}\text { Graphene nanoribbon (GNR) electronic bands, } \\
\text { Bloch states }\end{array}$ \\
$\omega=k_{\|} c / n_{g}=k_{\|} v_{g}$ & Graphene ideal dispersion \\
Light cone for a given (cladding) index & Energy $=E=\hbar\left(k_{\|}-k_{\text {Dirac }}\right) v_{\text {Fermi }}$ \\
$n: \omega>k_{\|} / n$ & Graphene cone (Fermi velocity) \\
$\Gamma$ point of Brillouin zone (minima, & $E>\hbar\left(k_{\|}-k_{\text {Dirac }}\right) v_{\text {Fermi }}$ \\
long-wavelength modes) & graphene) \\
Band of omnidirectional reflection & Dirac points of Brillouin $2 \mathrm{D}$ \\
Constant frequency contour (2D) & Energy band gap \\
Littrow diffraction, zone-edge mode coupling & Fermi surface (3D) /Fermi contour (2D) \\
Fabry-Perot modes (transverse) & Zone-edge degeneracy lifting \\
Grating diffraction efficiency in (back) reflection & Stationary electron states at $k_{\|}=0$ \\
Slow light (up to localization) & electron (back)-scattering efficiency \\
Monolithic system: photons are confined in a & Heavy electrons (up to localization) \\
given material & Monolithic device: electrons are confined in an \\
Grating teeth shape, grating phase portrait & engineered GNR \\
Mini-stopband, anticrossing of slow & Edge reconstruction shape, edge modifications \\
and fast modes & Electron notch filter (energywise) \\
"stripe of minigaps" & Electron comblike filter (idem) \\
Collective slow light, critical coupling & Selective comb filter (idem) \\
Photonic conductance & Ballistic conductance \\
\hline \hline
\end{tabular}

Studies of photonic crystal waveguides have been successfully carried out in $2 \mathrm{D}$, and we therefore argue here that this circumstance makes the analogy easier with GNRs. The third degree of freedom is best frozen thanks to a more classical guiding scheme, such as total internal reflection due to the index contrast in this third direction, as is well documented (see, e.g., Refs. 8-12). This vertical confinement plays essentially the same role as the single graphene sheet.

Interaction of propagating waves with periodic structures gives rise to coherent scattering, e.g., Bragg diffraction of $\mathrm{x}$ rays. For waves that are discretized by confinement in one or two of the three spatial directions, a quantitative account must deal with mode coupling. The coupled mode theory (CMT) is a workhorse of optoelectronics studies of periodic lightwave devices; it nowadays has innumerous applications.

It is especially interesting to compare zigzag GNRs (ZGNRs) with broad waveguides consisting of a dielectric core surrounded by two PhC claddings made of air columns drilled in the same dielectric [Fig. 1(a)]. This geometry is favorable for high-performance integrated devices. There is a small paradox here in the fact of considering periodicity in the cladding, whereas a GNR has periodicity built in its core - compare Figs. 2(a) and 2(b) — and a vacuum cladding. The main ingredient for our analogy however is in essence the interaction of basic waves in the core with the engineered periodicity along the edge, pictured in Figs. 2(c) and 2(d). These basic guided waves may notably have very simple underlying plane-wave decompositions. Then, to quite some extent, we shall see that diffraction of these plane waves on the edge is a correct vision of the main interaction step.
Confinement is nevertheless critical, sieving only discrete modes from a continuum. Hence, the diffraction efficiency of the guided wave then differs from that of an underlying plane wave: it is heavily modulated by the discretization of bands in these systems.

While our analogy focuses on the comparison with the \{dielectric core + air hole $\mathrm{PhC}$ cladding\} multimode waveguides, it does not preclude other possible analogies.

(a)

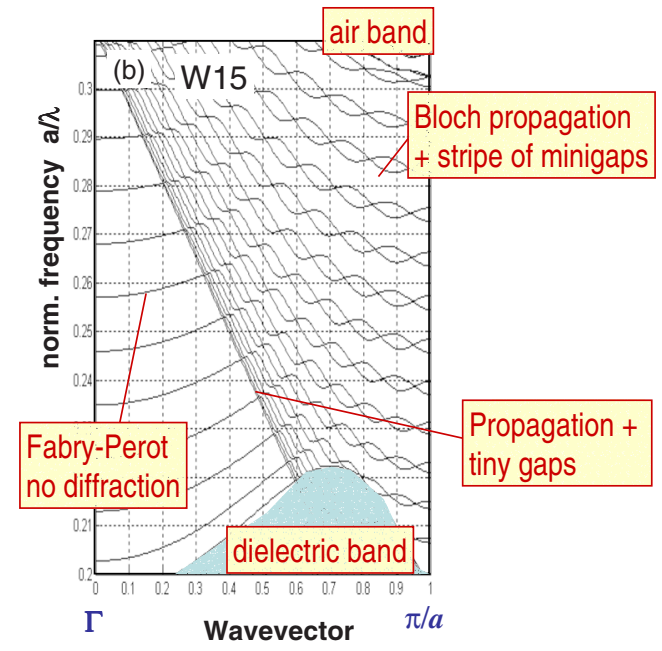

FIG. 1. (Color online) (a) Waveguide based on photonic crystal confinement, with air holes; (b) band structure for a waveguide consisting of 15 missing rows (W15) with its main features indicated, $n_{2}=3.21$, and an air fraction in the $\mathrm{PhC}, f \sim 35 \%$. 
(a)

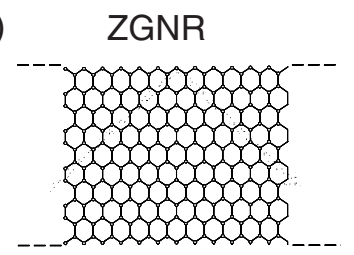

(c)
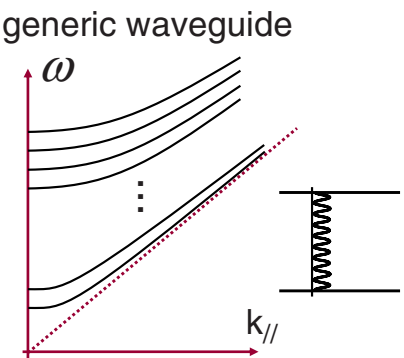

(d) mode coupling

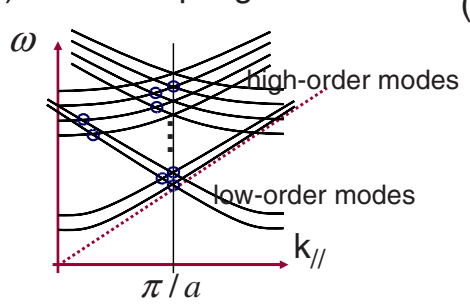

(e)

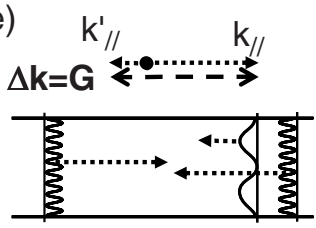

FIG. 2. (Color online) (a) Zigzag graphene nanoribbon; the light gray arrow represents wave vectors and local reflection; (b) the interaction with edge corrugation in the $\mathrm{PhC}$ waveguide; (c) dispersion of a generic uncorrugated waveguide, up to higher order modes, as sketched on the right of the graph; (d) Effect of periodicity, some interactions to be discussed (Littrow, near Littrow, and mini-stopband) have been circled; (e) picture of contradirectional mode coupling, either of different nature $\left(\left|k_{\|}^{\prime}\right| \ll\left|k_{\|}\right|\right)$or of same nature, the case of Littrow regime.

But with our choice, we find a consistent ensemble of mode coupling effects in GNRs, similar to those recently proposed and analyzed in the targeted category of $\mathrm{PhC}$ waveguides. To our knowledge, other guide systems have not been subjected to equally intense investigations concerning their internal mode coupling.

\section{B. Prospects prompted by the analogy}

Once the basis of our analogy is settled, we exploit it to propose different possibilities and functions in the frame of GNR-based electronic devices. The large degree of coherence and the observation of Fabry-Perot-type phenomena in single wall carbon nanotube (SWNT) (Refs. 1, 13, and 14) lead us to admit that the band structure largely governs transport phenomena in graphene and related systems, much as light in waveguides. ${ }^{13}$

We also assume that, sooner or later, it will be possible to modulate a GNR potential periodically at the $\sim 1-10 \mathrm{~nm}$ scale, selectively on its edges. This may demand nanometercontrolled systems, such as arrays of SWNT, metal-like fullerene assemblies, "peapod" variants of fullerene, etc., seemingly out of reach today but that can be envisioned given today's pace in related nanotechnologies. The demand on accuracy of potential modulation restricted to the sole edge region may ease the challenge because no $2 \mathrm{D}$ uniformity is required.

The particular phenomena that we propose to use essentially select discrete electron energies across broad momentum distributions. To this end, they involve either a single dispersive band with well defined minigaps (also called ministopbands or mode gaps), or a plurality of bands that are remarkably flat within a substantial fraction of the Brillouin zone. The corresponding optical phenomena thus fall in the category recently coined as slow light phenomena.

One first case is a notch filter for electrons, which may reject narrow energy windows. It is based on the selective diffraction of the GNR fundamental (extended) mode by the periodicity, this diffracted power being heavily modulated by the confined fate of the diffracted wavelets. Only wavelets fitting the momentum discretization may deplete energy from the fundamental mode and affect its momentum, hence the electrical current in the GNR. One may then expect specific dips in the transmission. Equivalent phononic ${ }^{15}$ and photonic phenomena have been recently documented, notably in the author's group under the name of mini-stopband, as further discussed later ${ }^{16-19}$ in relation to the generic Fig. 1(b) presenting the dispersion relation of a broad $\mathrm{PhC}$ waveguide.

A second case, somewhat more challenging, is analogous to a phenomenon that we recently identified under the term of Littrow lasing in broad PhCs waveguide. ${ }^{20}$ We furthered this concept by a theoretical study ${ }^{21}$ and by an experimental test of the heavily modulated photonic DOS. ${ }^{22}$ The core of this effect is the ability of diffraction at the guide edge to localize light along the waveguide, notably if diffraction scatters waves back,. This case is known as Littrow configuration in optics, and much used in spectrometer (blazed gratings) and external cavity lasers. The advent of Littrow diffraction in broad waveguides results in the formation of "stripe of minigaps" [seen in Fig. 1(b)], whereby bands at the Brillouin-zone edge take a global form of a set of hyperbola. The bands are now squeezed between these stripes, as a result of all forward modes being coupled to all backward modes (within symmetry allowance). For a proper coupling value (a large value relative to the energetic band separation, and logically a high equivalent diffraction efficiency), the bands become very flat in a relatively extended region of the band structure, spanning a number of the interacting branches sketched in Fig. 2(d), and flattening them. We believe that this can be exploited to produce narrow energy electronic transmission windows for transport in GNR devices.

Furthermore, assuming ballistic transport, we shall propose open resonators based on this phenomenon. One of their interests, based on ongoing evolutions of microresonators in optics and in photonic crystals ${ }^{23}$ would be the ability to implement an electronic version of a Fabry-Perot (FP) resonator, e.g., with narrow peaks in transmission mode, but without having to physically traverse any "contact" or "barrier" (mirror) layer. More cascaded electronic devices could then be more easily envisioned, allowing ultimately the exploration of geometries much richer than the popular electronic islands often fabricated for recent mesoscopic transport studies. 
We note that several theoretical works, $2,14,24-29$ have already proposed analogies between photonic crystal waveguides and GNRs. They relate to different, and generally more sophisticated aspects of transport, to specificities of particle description and of electron-hole pictures in graphene (e.g., valleys), which are not our direct purpose here. We nevertheless anticipate that the potential of the present photonic analogy combined with the powerful tools for description of charged particles in graphene opens vast perspectives.

It may also be useful to remind the reader that in optics, whereas the multimode nature was primarily considered to plague device use, causing dispersion, etc., multimode waveguides (rather than waveguide arrays) were nevertheless of interest in the frame of recent studies of photonic implementations for fundamental phenomena (localization, ${ }^{30,31}$ assisted tunneling ${ }^{32}$ ) or in relation with clever interaction schemes. $^{33}$

In the following, Sec. II outlines the ZGNR tight-binding calculation, in the spirit of Ref. 7. Section III provides results from ZGNRs with various superperiods and various periodic modulations of the edge atom potential. Simple choices shown here are aimed at feeding, in Sec. IV, the basic analogies between PhC waveguides and ZGNRs: mini-stopbands, and role of edge symmetries and of superperiod. In Sec. V, we apply the concept of flatbands induced at a critical coupling condition and observe the formation of minigap stripes in graphene and ZGNRs, the counterpart of the analog photonic phenomenon. In Ref. 22, we had only hinted at the unnoticed appearance of the minigap stripe in the reconstruction study of Ref. 6 and to the relation with the superlattice concepts of Ref. 34. Section V concludes these studies.

\section{TIGHT-BINDING CALCULATION}

We define a supercell for TB band-structure calculation in a ZGNR as outlined in Fig. 3, and in the spirit of Cresti et al.'s ${ }^{7}$ approach. The basic unit cell we use has four atoms. The interatomic dimension is $a_{0}$ and the four-atom unit-cell size along the GNR is $a_{z}=a_{0} \sqrt{3}$. The supercell tiles $N_{x}$ cells in width and $N_{z}$ cells along the small dimension, along the ZGNR. The superperiod is thus $N_{z} \sqrt{3} a_{0}$ and there are $N_{\text {at }}$ $=4 N_{x} N_{z}$ atoms in a supercell. The TB-related dispersion can be computed simply by setting up the proper matrix elements among the $N_{\text {at }} \times N_{\text {at }}$ atom pairs. Here, the translation operator only imposes a $k_{\|} \equiv k_{z}$ value. The matrix element of the TB Hamiltonian between neighbor atoms $m$ and $n$ simply reads $h_{m n}=E_{0} \delta_{m n}+t \exp \left(i k_{\|}\left[x_{m}-x_{n}\right]\right)$, where $E_{0}=0$ is the convenient energy of the nonbonded carbon atom and $t$ is the standard (per bond) tight-binding energy, so that the overall energy spans $\left[E_{0}-3 t, E_{0}+3 t\right]$. Depending on the matrix size and desired eigenmode range, we use "full" or sparse matrices (under MATLAB®). The simplest computation providing a ZGNR band structure is for $N_{z}=2$, and is thus folded with respect to the canonical representation.

The simplest modifications of the ZGNR consist of offsetting atom energies to some specific values $E_{0}>0$ for selected edge atoms, either abruptly or gradually starting a few atoms away from the edge. Atoms at the edge can also be merely removed.
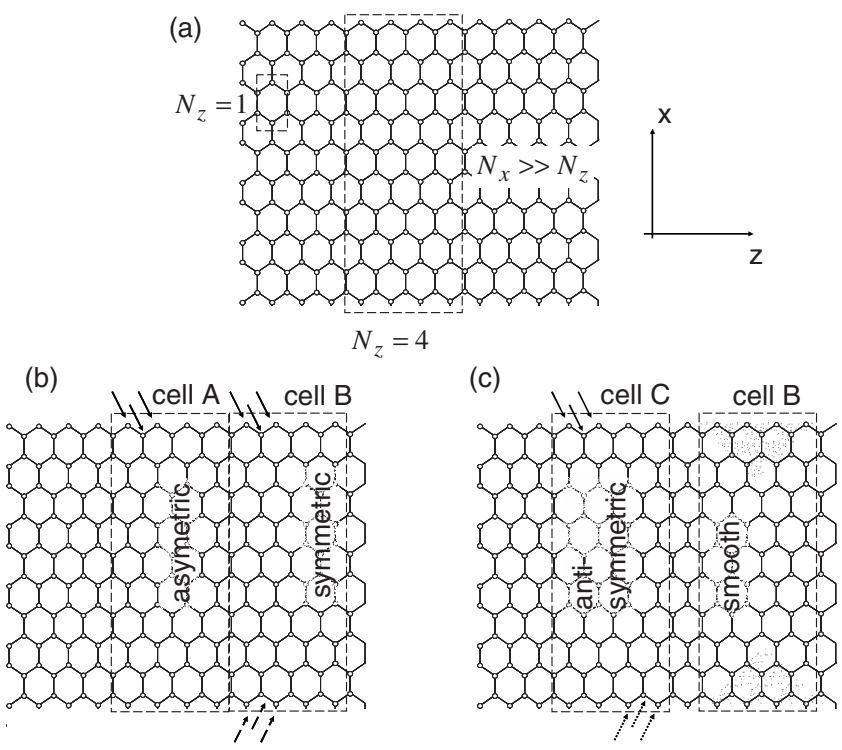

FIG. 3. (a) ZGNR with a graphene rectangular unit cell and with supercell definition; (b) supercells with modified atoms (pointed by small arrows) of asymmetric type (cell A) or of symmetric type (cell B); (c) supercell with antisymmetric modification (cell C) or smooth symmetric modification (cell B), the gray triangles indicating the imposition of some local bias to the atoms only in these areas.

Due to folding, the Dirac point lies at some fractional position of the supercell BZ for values such as $N_{z}$ $=2,4,8,16$. For $N_{z}=3,6, \ldots$, the Dirac point lies at the very zone center. If no atom is modified or removed, the supercell of course results in a mere folding, in analogy with the empty-lattice band pictures. The introduction of modified edge atom potential affects the band structure in those regions where folded modes cross unfolded modes.

The generic effects are sketched in Fig. 2, with the Dirac point taken as the origin and only positive energies are shown. Starting from a basic dispersion [Fig. 2(c)], the folding causes crossings either between modes of similar nature or of more different nature, e.g., fundamental and higher order modes in Fig. 2(d), with some coupling pointed out by circle markers. Figure 2(e) shows a real-space picture of this coupling, together with momentum conservation. These simple rules hold for both systems and will allow us to carry on the analogy in more detail below. We calculate the bands for the various configurations discussed below, in order to assess the depth of the analogy with broad photonic crystal waveguides.

\section{GENERIC EFFECTS OF ZGNR EDGE MODULATION}

We first present in Fig. 4(a) the basic folded known ZGNR band structure as a reference. Here we used $N_{x}=50$ and $N_{z}=2$ (400 atoms). As said, it is folded at the specific BZ edge, in this form of TB calculation. The Dirac point lies at two-thirds of the BZ and the waveguide-like bands can be recognized, with their locally hyperbolic shape.

At high energies, the nearest-neighbor (NN) TB calculation is not accurate. However to describe the basic Floquet- 


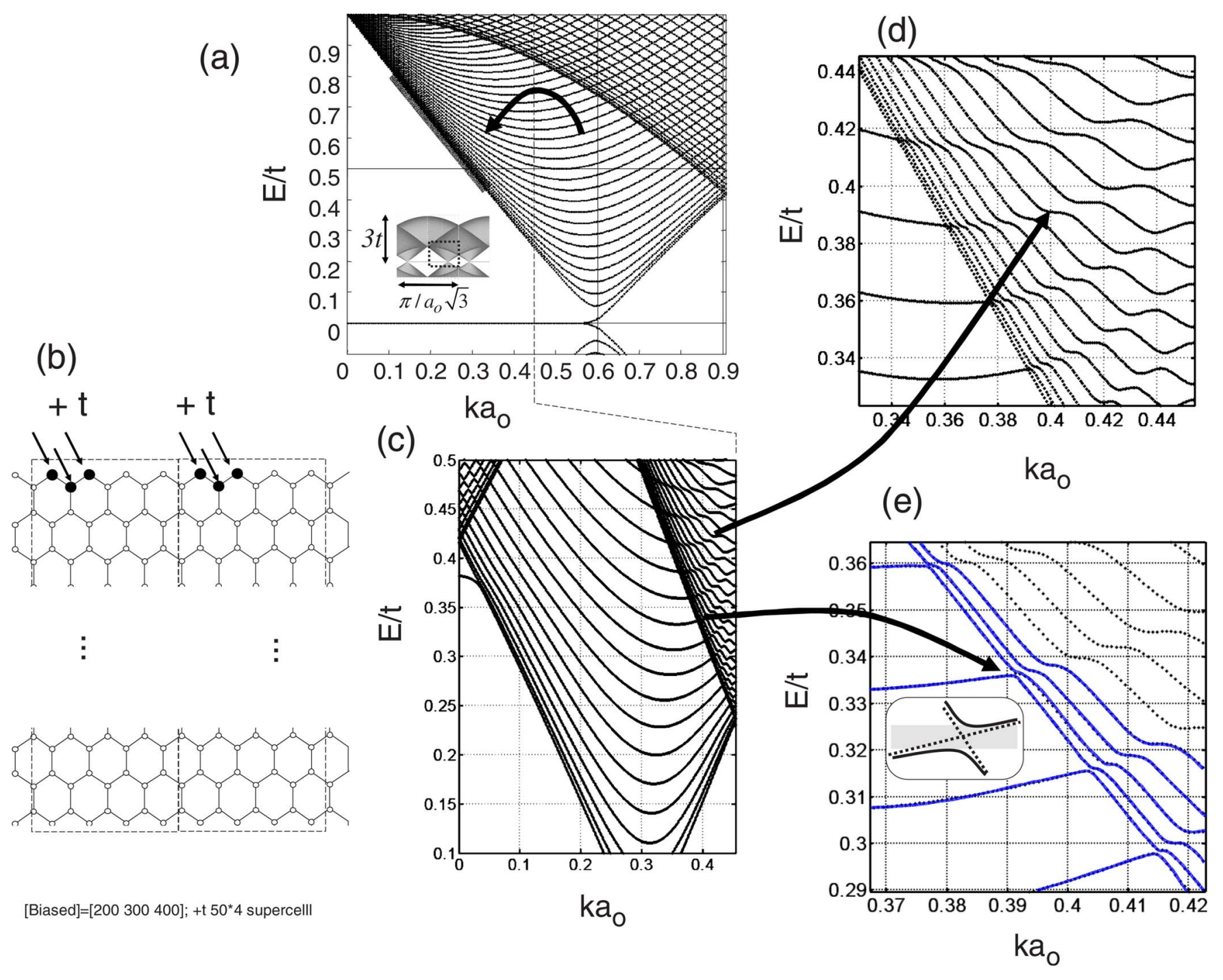

FIG. 4. (Color online) (a) ZGNR dispersion relation for a $50 \times 2$ supercell (400 carbon atoms), normalized to the tight-binding parameter $t$; the fundamental mode is underlined by a gray thick line; the inset shows the band structure repeat pattern spanning $\left( \pm \pi / 2 a_{0} \sqrt{3}\right)$ $\times( \pm 3 t)$; (b) edge modification by an excess bias $+t$ of the three indicated atoms of a $50 \times 4$ supercell ( 800 atoms), whose dispersion relation would otherwise be the folded version of (a) as indicated; (c) small energy region of the dispersion of modified ZGNR; (d) stripe of minigaps starting to form around zone edge; (e) small anticrossings (mini-stopbands) affecting the fundamental and few next modes.

Bloch modes that are likely to participate to conduction and that are essentially endowed with a constant Fermi velocity in an unconfined graphene sheet, we believe that NN TB is sufficient. We present our data in normalized units of $t$ throughout, remaining universal enough.

Next, as shown in Fig. 4(b), we consider the change introduced by a modulation of the potential of $E_{0}=+t$ instead of $E_{0}=0$ in three of the eight edge atoms of a superperiodic ZGNR with $N_{x}=50$ and $N_{z}=4$ (800 atoms). This twice larger cell now induces a folding at $k a_{0}=\pi / 4 \sqrt{3}$.

In general, an edge modification may take the form of a mere carbon atom removal, or a change in energy, which decouples gradually the biased atoms from their unbiased neighbors. Any such modification introduces anticrossings in the indicated conduction-band region of Fig. 2(d), near the zone edge. Of course, the formerly dispersionless state at zero energy becomes here a slowly varying branch. This is logical since the modification disrupted the singular proper- ties of the GNR edge atoms causing this special branch.

The results are shown in Figs. 4(c)-4(e). For the ZGNR fundamental modes and the few first-excited modes (all folded above $E / t \sim 0.24)$, only tiny gaps, regularly spaced, decorate the original dispersion [Fig. 4(e)]. The wavy pattern in Fig. 4(d) will be analyzed as a stripe of minigaps in the next section.

Next, we introduce a symmetrical perturbation, with twice more atoms affected, at both edges instead of a single edge, as shown in Fig. 5(b), with the same supercell of 800 atoms. The band structure, Fig. 5(a), now takes the shape of intertwined necklacelike branch pairs. Every other anticrossing is forbidden, and crossings take place instead. Thus, the tiny gaps on the fundamental branch and first-excited modes are now formed only at every other mode crossing. We had pointed out in Ref. 22 that a similar phenomena occurred, albeit unnoticed, in recent reports of periodically modulated GNR due to edge reconstruction, ${ }^{6}$ and could also arise due to 


\section{(a)}

岀

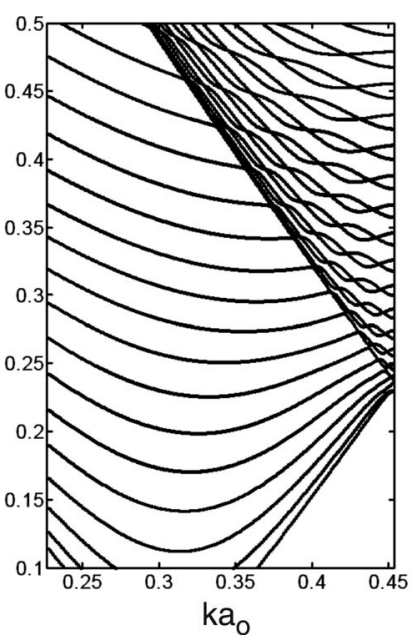

(b)

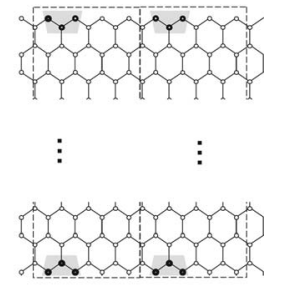

FIG. 5. Dispersion relation (a) of a $4 \times 50$ ZGNR (800 atom cell) with symmetric edge modification of $3+3$ atoms by $+t$ shown in (b). Note the paired bands resulting in more clear stripe of minigaps. The antisymmetric configuration gives the same pattern, with paired band degeneracies located at zone edge rather than the situation here.

mesoscopic regular undulations ${ }^{34}$ of the graphene sheet.

We checked that, upon calculating an antisymmetric rather than a symmetric structure (for example, by having the modified atoms at each edge shifted by a half period), the overall scheme is the same but the pattern of interaction is the alternate of the previous one. The glide/mirror symmetry induces a $\pi$ phase shift in the relevant perturbing potential's Fourier components. ${ }^{35}$

The same kind of perturbations may also be applied to the case of the longer superperiod $N_{z}=6$ [Fig. 6(a)]. For such multiples of three, the crossings of interest now fall at the $\Gamma$ point of the supercell Brillouin zone. The results [Fig. 6(b)] follow the same trends for single-sided or symmetric varia-

(a)

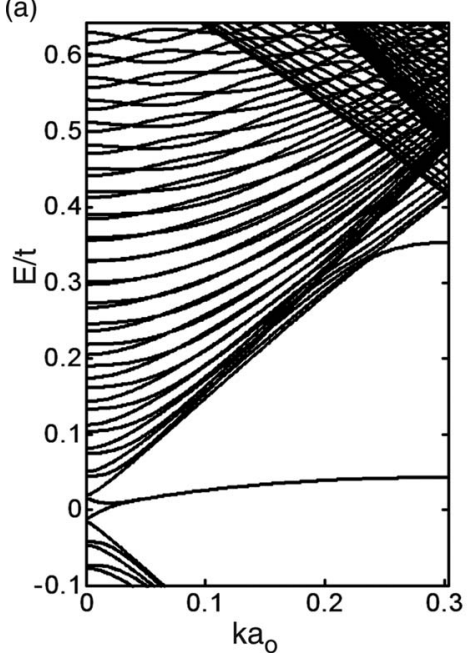

(b)

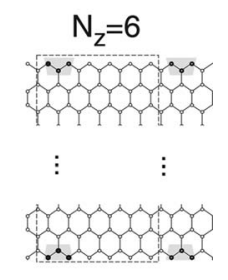

FIG. 6. Dispersion relation (a) of a $6 \times 50 \mathrm{ZGNR}(800$ atom cell) with symmetric edge modification of $3+3$ atoms by $+t$ shown in (b). For this superperiod, Dirac points are brought back to $\Gamma$ point.

tions but it is not as easy to distinguish various regimes as it was in Fig. 4.

\section{ANALOGIES FROM PHOTONIC CRYSTALS TO GRAPHENE NANORIBBONS}

These first results are in clear analogy with band-structure features of broad Wn waveguides defined in a triangular lattice of holes in a dielectric matrix, as reminded in Fig. 1. Typical refractive indices for such matrices are in practice taken as the effective indices of semiconductor heterostructures. For example, one has $n_{\text {eff }} \sim 3.21$ in the InP-based cases common in telecom optoelectronic devices. A lower value $n_{\text {eff }} \sim 2.8$ applies to popular semiconductor membranes (silicon, GaAs, InP). This quantity $n_{\text {eff }}$ best describes the fate of waves in a two-dimensional view. It is well suited to the graphene sheet analogy that we want to carry out here. The analogy does not require, on the contrary, identifying air holes with carbon atoms. Rather, the ingredients are the photonic guided waves in the unpatterned heterostructure (seen as scalar in the most simplified instances), and the FloquetBloch electronic modes of graphene sheet. Then, the ZGNR edge is well mimicked by a $\mathrm{PhC}$ patterning. Photons/ electrons are forbidden to leave the guide/ZGNR medium, constituting a periodic boundary on which diffraction in a few discrete grating orders is allowed. The local description of the interaction of plane waves with periodic boundaries has its usefulness but we believe that the analogy between modes encompassing the whole system is better suited to the analogy and presently richer.

We start here by the region occupying the largest fraction of the band structure, from the BZ edge on. We examine next the case of the fundamental mode, as it is a less general case in terms of phase-space volume.

The optical situation known as the Littrow mount is analogous to the Brillouin-zone-edge situation; it rests on exactly the same $k_{\|}$wave-vector configuration, and periodicityinduced coupling. From the generic case of Fig. 2(e), we now have the ray/wave pictures of Fig. 7, whereby guided modes are identified through their two Cartesian $k$ components. Exact zone-edge situations amount to exact Littrow diffraction (back reflection) events, with the anticrossing lifting the perfect degeneracy of these points. Near the BZ edge, anticrossings involve guided modes of different quantum number but not too different. This can be termed "nearLittrow" diffraction. The general resulting situation is what we have termed a stripe of minigaps, ${ }^{22}$ appearing clearly in Fig. 1 on one hand, and in Figs. 4(d) and 5(a) on the other hand. This accumulation of zero DOS minigaps means a sizable DOS reduction in the concerned region of $k$ space. In the optical regime, we have recently been able to visualize a strong DOS modulation associated with these stripes thanks to a specially designed experiment. ${ }^{22}$ We elaborate on ways to exploit this further below, going to a "critical coupling regime" 21 (already apparent in Fig. 6 but this case is not so analog to Fig. 1 then).

In terms of band structure, the ZGNR phenomena identified above are thus completely analogous to those met in guided modes of a broad $\mathrm{PhC}$ waveguide. We note that, even 
Ray picture : Littrow diffraction
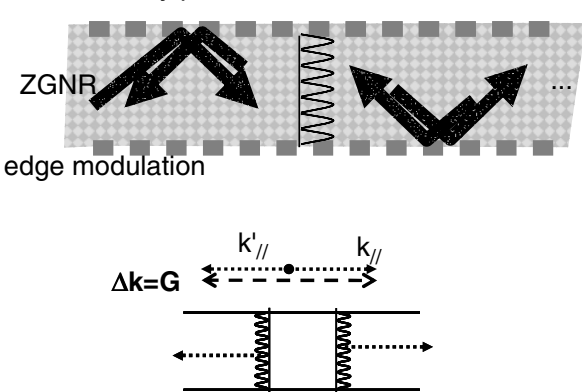

wave picture : zone edge diffraction

FIG. 7. Ray and wave pictures of the role of ribbon edge corrugation. In the ray picture, Littrow diffraction occurs and a roundtrip phase condition between edges is needed to build up Bloch modes that eventually propagate in spite of this endless backward diffraction; in the wave picture, counterpropagating modes interact due to the wave vector $G$ brought by the corrugated edge period, the interaction inducing a zone-edge splitting with forbidden propagation inside the minigap, and band formation around.

on the optics side, these phenomena are not specific of PhC: the same occurrence of mode anticrossing also arises for simply corrugated waveguides, when guided waves explore the Littrow regime. ${ }^{21}$ In canonical $\mathrm{PhC}$ systems, symmetries naturally appear so that modes couple in pairs of branches as seen here in Fig. 1(b). Also, specific to PhC and metal-clad waveguides, confinement exists for any wave vector, whereas it may be quite compromised in ordinary refractive optical waveguides for modes below the cladding light line reading $k_{\|}=n_{\text {cladding }} \omega / c$.

Let us now discuss the coherent interaction of the fundamental modes with higher order modes. This interaction is manifested by minigaps signaled in both cases [Figs. 1 and 4(e)]. It corresponds to what is termed by some as mode gap, and by us as mini-stopband: a local stop band in the band structure. This mini-stopband is valid for the few fundamental modes, arising when the periodicity is sampled in such as way as to couple them with a series of slower counterpropagating higher order modes. ${ }^{18,19,36}$ In the photonic case of Fig. 1 and in high-frequency limit cases $(u>0.3$ in Fig. 1), these modes have a vanishing wave vector and become just normal-incidence Fabry-Perot modes.

In view of the above, the general issue of anticrossing effects vs edge modulation potential may be seen to carry a strong analogy with the widely addressed engineering issue of optimizing diffraction efficiency from gratings: the choice of corrugation profile (here of edge potential) determines how the spatial harmonics are addressed and eventually excited from the interaction with unperturbed modes. It is not very common for partly confined electronic state to consider diffracted orders but the exercise is the same, in essence, as the common electronic diffraction studies, such as the low energy electron diffraction (LEED) and its descendents (by the way, electronic diffraction received vast contributions from J. Pendry, ${ }^{37,38}$ who became a key contributor to the area of periodic photonic structures before his more recent famous contributions to photonic and metamaterials).

Prompted by this idea and by our recent work on slow light and stripe of minigaps, ${ }^{22}$ we try in the following to

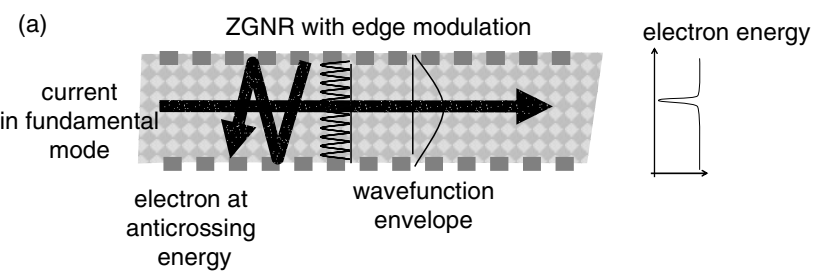

(b) open resonator

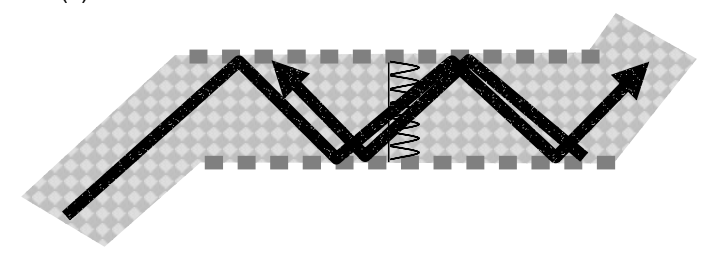

FIG. 8. (a) Expected effect of the interaction between fundamental mode and higher order mode pictured in Figs. 2(e) and 4(e): notch filtering of electrons of a fairly precise energy; (b) open resonator geometry exploiting strong diffraction and slow modes arising for increasingly wide stripe of minigaps; the electron is always retained well inside the graphene, and does not have to cross any barrier but may nevertheless exhibit Fabry-Perot behavior for round-trip resonant conditions.

verify the same trend for modified ZGNR, a trend not hitherto reported to our knowledge. Before doing so, we elaborate on transport properties that may be expected (Fig. 8) from the above. We may notably infer that if electrons are transported in the fundamental ZGNR mode [Fig. 8(a), the envelope of this mode has a single lobe across the ribbon width, whatever the local atomic amplitudes inside the unit cell], the mini-stopband may act as a notch filter in the electronic energy domain. Such filters are not obvious to implement in vacuum electron optics, nor with resonant barriers/ wells inserted across the electron path. They would require coupling to localized states defined aside the ribbon, for example. Such approaches seem far less convenient to implement than the simple use of a ZGNR with a controlled boundary potential although this latter solution is, admittedly, at the limit of what can be envisioned.

The electrons not transmitted in such a filter would be ideally reflected but all the extra scattering channels would blur this picture, and they would eventually participate to a background leakage current. Device design and exact scattering conditions in the ZGNR would then dictate the relative strength of the main (ballistic) current carried by the fundamental ZGNR mode, and the (thermalized) multimode valley current. Quantum transport treatments are necessary to get a full picture, absent in the standard photonic (bosonic) case. We note that converse attempts to define "photonic conduction" in PhC-based waveguides have been recently reported. ${ }^{39}$ They rather result in standard expected scaling laws (one conduction quantum per effective half-wavelength width) rather than in the singular behavior of the ministopband expected here. This is not contradictory, given the difference that exists between our $\mathrm{PhC}$ and the dielectric column $\mathrm{PhC}$ with air background of Ref. 39. This latter case support guided modes that do not undergo coupling to any other mode thanks to the intrinsically limited magnitude of the wave vector in an "air guide." 


\section{FLATBANDS AND MINIGAP STRIPES BY SIDE POTENTIAL, DOS}

For a ZGNR that is both wide enough and with a long enough period, the region around the band folding is situated at a modest electronic energy $(E=0.2 t$ or less). This region evokes a basic picture of a large set of modes, with similar parallel dispersion curves, crossing their folded counterparts at the Brillouin-zone edge. The abundance of crossings in this case calls for a different analysis as compared to the standard Bragg reflection in a single mode waveguide. We have shown in Ref. 21 that, when the coupling constant between modes of the uncoupled basis reaches a sufficiently high value, these anticrossings merge. They form a stripe of minigaps, also seen experimentally ${ }^{22}$ in $\mathrm{PhC}$ waveguides. Additionally, around a precise critical value of the coupling, ${ }^{21}$ the branches delimiting these stripes of minigap loose most of their wiggles and adopt a much smoother curvature. Such an effect arises (unintentionally) in the middle of Fig. 6, where the necklace branches stick together, but again this geometry is not easily mapped onto the known photonic ones. More generally and heuristically, very efficient Littrow diffraction takes place at this stage. We are then in a resonator situation largely analogous to the Fabry-Perot case but accommodating the specific slanting effect of the Littrow reflection pair in a guide. [Figs. 7 and 8(b)]. Specifically, the flat branches acquire a locally hyperbolic shape, much as basic FP modes.

In Ref. 22, we showed that, even at the intermediate steps well before flatbands, a deep DOS modulation did arise from the stripe of minigaps, a feature usually thought to be washed out in a broad waveguide. In our graphene ribbon, we have attempted to reproduce this situation because of its potential interest: by modulating only the edge of the ribbon, we may render a large region of $k$ space much less "conductive" if we manage to slow down the electron wave packets in it. A reasonable guess is to employ a partially ballistic operation regime for carriers (electrons) whereby the system is made very sensitive to edge potential modulation: in a ballistic regime, injection under the proper angle, shown in Fig. 8(b), may suffice to address a restricted portion of band structure $(\sim 10 \%)$, much as it does in optics, whereas in a more diffusive regime, one thoroughly samples the Fermi surface of the system (a Fermi contour in 2D graphene). All bands at a given energy then participate to transport, a situation that somehow relates to the way spontaneous emission samples available states in photonics, e.g., in a photonic wire. ${ }^{36}$

Figure 8(b) suggests an open resonator geometry exploiting only three reflections when we adopt the ray picture. This may suffice to get a resonance, based on the flatbands at critical coupling. This open resonator geometry has the same kind of advantages as discussed earlier: it uses "monolithic" electron ballistic propagation, and requires no tunnel barriers through foreign materials (be it vacuum) to couple in and out of the resonator due to the intrinsic reflective nature of its constituent, and to the selection of a single possible diffraction order at lower energies. This arrangement should provide a fair immunity to the undesirable atom-scale chemical environment, whose disturbance is far more likely in tunnel sections than in "bulk" graphene sections. It thus constitutes
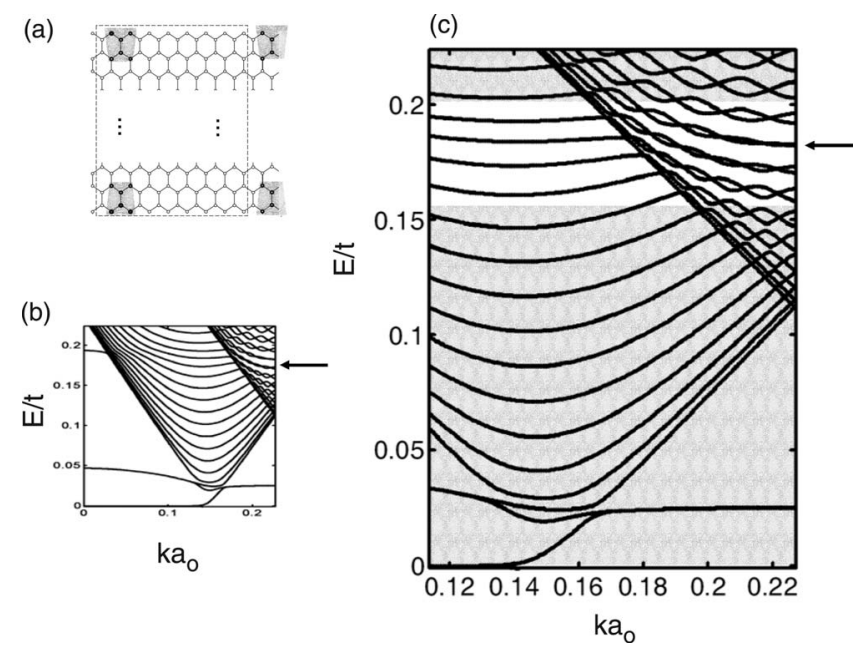

FIG. 9. (a) Six atoms on each side of the ZGNR are biased to the same potential (gray area); (b) resulting band structure for $V_{\mathrm{Bias}} / t=0.48$; an arrow indicates the perturbed energy region; (c) zoom on the perturbed energy region showing very flat bands and locally hyperbolic stripes of minigaps.

a key point toward large-scale applications. We are not aware of other electronic resonator proposal with similar advantages. The intrinsic good properties of graphene (large Debye temperature and room for small electron phonon coupling) could then be exploited in nanoelectronic active devices (transistors, electron valves, etc.). The spin parameter was not considered here but it could still add extra opportunities.

Still using $t$ as the natural energy unit, we found that a bias of some edge atoms of the order of $0.5 t$ is sufficient to repel lower energy electrons almost fully at the atomic scale. Then, our effort amounts to shaping a grating (a bias landscape) with maximal Littrow diffraction efficiency. The use of a photonic analogy may be of some help to shape a potential that diffracts an electronic wave. One may infer that the crystalline nature of graphene could be averaged to recover a continuous tooth profile (in grating terminology, a reliable microscopic phase portrait could be devised). At our smaller numerical simulation scale, however, we are constrained to use modest periods $\left(\sim 10 a_{0}\right)$ because we want to retain the multimode aspect related to the large GNR width. The discrete atomic arrangement of graphene may then still play a role (as it does for reconstruction of GNR edges ${ }^{6}$ ). This limitation pushes us away from the "long-wavelength regime." It is somehow an unfortunate circumstance not only to further our analogy but also because smaller electronic energies, smaller bias modulations, and larger teeth periods would be preferred technological options for a real-world implementation of very low-energy switches.

We nonetheless find analogous results. We restricted the modification to a pair of six clustered atoms on each side of the ZGNR, three atoms at the zigzag boundary, and their three neighbors inside the ribbon [Fig. 9(a)]. We set the potential of these twelve atoms at some given value $V_{\mathrm{Bias}} \leq t$ and plot the results as a function of $V_{\mathrm{Bias}} / t$.

In Fig. 9(b), we show a view of the band structure for $V_{\text {Bias }} / t=0.48$, encompassing a Dirac point. We magnify the region of interest for flatbands in Fig. 9(c). Of course, the 
GNR edge state is also perturbed by this modification but we do not investigate this here. Rather, we note that the perturbed energy range is localized around $E / t=0.17-0.19$. It can notably be seen at the smaller $k a_{0}$ values, causing an attractive shift of some modes on the left of Fig. 9(b), at $E / t=0.19$. A single localized mode also exists below the graphene cone bounded by the fundamental mode [Fig. 4(a)].

The main feature, in our view, is the clear advent of flattened bands and associated stripes of minigaps on the left of Fig. $9(\mathrm{c})$, at $E / t \approx 0.18$, causing a regime of very limited group velocity. Two bands here are especially flat: one pointed by the arrow and the one below.

As we have not designed our periodic potential so specifically, we anticipate that the phenomenon is general enough, i.e., it can equally appear with any other potential that respects symmetry and has a sufficient global strength. Attempts that are not shown here confirmed this trend.

There are also differences when comparing to the broad photonic corrugated waveguide case of Fig. 1. Here only two bands are really flat while the stripes of minigaps tend to shrink rapidly at higher or lower energies. This energetic localization of the flatter band phenomenon to a somewhat narrow interval does not occur on the photonic side of the story (Fig. 1) or not to the extent seen here. We hypothesize here that this distinction possibly stems from the fundamental difference of Maxwell and Schrödinger equations with respect to the potential-energy term. This term is fixed for Schrödinger equation, whereas, in known equivalences of Maxwell (or Helmholtz) equations, the potential term, being typically of the form $\omega^{2} \Delta \varepsilon$, indefinitely increases with frequency (see also Ref. 40). This might explain retrospectively the persistence of marked density-of-state features over very wide frequency ranges in photonics. ${ }^{22}$ In the electronic case, the incident energy has to match that of the specific states added by the perturbation to ensure a resonant scattering cross section and a maximal disturbance. Otherwise, the bands turn back to the nonmodulated case because the larger electron kinetic energies are no more balanced by a matched term.

To eventually substantiate the amount of dispersion slow down that can be obtained based on our very simple electronic grating design, we plot a partial electronic DOS defined below as a color map, as a function of $V_{\mathrm{Bias}} / t$ along the abscissa, and as a function of normalized electronic energy $E / t$ on the ordinate. This is done in Fig. 10, with a hot color map and a linear DOS scale in arbitrary units. The partial electronic DOS is a way to visualize the expected effect of ballistic transport in conjunction with some $k$ relaxation due either to collision or to the nature of the wave packet itself, e.g., a spatially partly incoherent electronic wave.

To fabricate these partial DOS maps, we specifically made band-structure calculations for a range of values of $V_{\text {Bias }} / t$, using $600 k$ points along the reduced Brillouin zone. We then selected either the 50 or 100 leftmost states of the set [with reference to Fig. 9(c)], i.e., those with the larger $k$ values, around $k a_{0} \sim 0.18-022$ in the present case. These choices amount to collect $8.3 \%$ and $16.7 \%$ of the total number of states.

It is clear that, for partial DOS for the 50 states only, we can see a very nice DOS extinction between allowed bands,

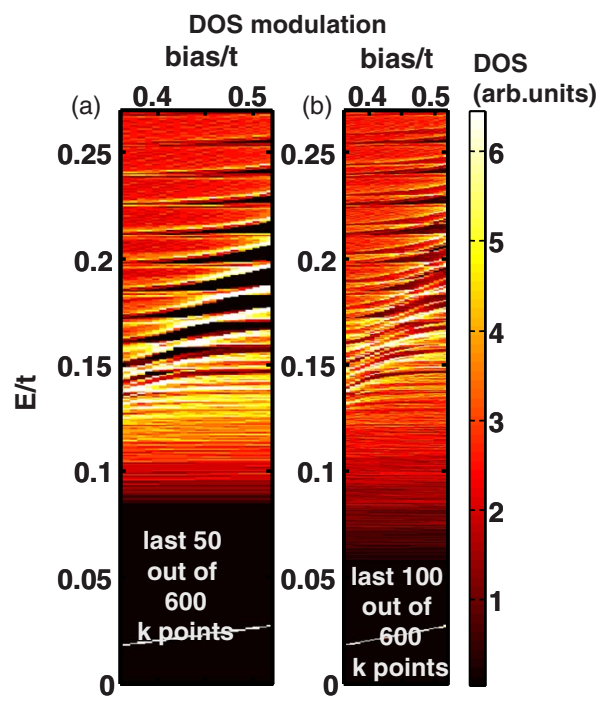

FIG. 10. (Color online) (a) Color map of the density of states for the leftmost $k$ values $\left(k a_{0} \in[0.2078,0.2267]\right)$ of the band structure of Fig. 9, for variable potential $V_{\text {Bias }} / t$ as indicated; (b) same DOS color map [not normalized to (a)] but gathering now the twice broader range $\left.k a_{0} \in[0.1889,0.2267]\right)$

at $E / t$ values around typically $0.15-0.2$. This is all the more striking because the $8 \%$ of the states probed are much more than the fraction $12 / 3600 \sim 0.3 \%$ of atoms whose potential was affected. This leverage is a key aspect of using a regime of coherent electron diffraction.

Of course, we cannot affect all the states. If we enlarge the DOS to collect 100 of 600 states, the gaps are almost disappearing. But from the color map, a large modulation does persist.

Calculations on about 100000 atoms instead of 3600 here would be needed to bring the energy range to well below $E<0.1 t$ [hence only a few thermal energy quanta $k_{B} T$ (with $k_{B}$ the Boltzmann constant, and $T$ the temperature), as often desired in devices] while still retaining a largely multimode regime.

Still, at such a size, with the best mean-free paths reported at room temperature being larger than $3000 \mathrm{~nm}$, there is enough room to sustain a dozen reflections in a 50-100-nmlarge ZGNR. This would result in an equivalent finesse of the transmitted electronic waves of more than 30. In other words, the device of Fig. 8 would transmit $<3 \%$ of the electronic energy range targeted by this effect and reject the other $97 \%$.

\section{CONCLUSION}

We have proposed a fruitful analogy between photonic crystal broad waveguides and graphene nanoribbons, on account of the linear dispersion of electrons or photons in the guiding medium. The analogy is substantiated by several tight-binding band calculations that confirm the applicability of the mode coupling effects known from the photonic side. The same effects do arise in nanoribbons at low energies if the GNR edge has a periodic modulation of a couple of nanometers. We have more precisely investigated modifications 
of edge atom potentials. We found several key effects previously reported in photonic crystal waveguides, most notably the mini-stopbands and stripes of minigaps. We have proposed ways to exploit the strong and specific electronic reflection at the Brillouin-zone edge to control a quasiballistic device conductance. The principle of Littrow diffraction, completely analogous to diffraction at Brillouin-zone edge, can be applied to electrons, and may reveal elegant and useful effects. We hypothesized that some remaining differences were due to the rather profound difference between Schrödinger equations and Helmholtz-type equation, where the potential term follows the frequency. Overall, we hope that our remarks will prompt new schemes for the beneficial use of GNRs, in particular making them functional and immune to environmental disturbances. We also hope that they could find some echoes in acoustics or in any domains where wave behavior in a multimode one-dimensional arrangement provides the dominant mechanism.
${ }^{1}$ W. Bishara, G. Refael, and M. Bockrath, Phys. Rev. B 78, 165405 (2008).

${ }^{2}$ K. S. Novoselov, A. K. Geim, S. V. Morozov, D. Jiang, Y. Zhang, S. V. Dubonos, I. V. Grigorieva, and A. A. Firsov, Science 306, 666 (2004).

${ }^{3}$ J. D. Jackson, Classical Electrodynamics (Wiley, New York, 1999).

${ }^{4}$ Y. W. Son, M. L. Cohen, and S. G. Louie, Phys. Rev. Lett. 97, 216803 (2006).

${ }^{5}$ K. Nakada, M. Fujita, G. Dresselhaus, and M. S. Dresselhaus, Phys. Rev. B 54, 17954 (1996).

${ }^{6}$ P. Koskinen, S. Malola, and H. Hakkinen, Phys. Rev. Lett. 101, 115502 (2008).

${ }^{7}$ A. Cresti, G. Grosso, and G. P. Parravicini, Phys. Rev. B 76, 205433 (2007).

${ }^{8}$ J.-M. Lourtioz, H. Benisty, V. Berger, J. M. Gérard, D. Maystre, and A. Tchelnokov, Photonic Crystals, Towards Nanoscale Photonic Devices, 2nd ed. (Springer, Heidelberg, 2008).

${ }^{9}$ H. Benisty, D. Labilloy, C. Weisbuch, C. J. M. Smith, T. F. Krauss, A. Béraud, D. Cassagne, and C. Jouanin, Appl. Phys. Lett. 76, 532 (2000).

${ }^{10}$ S. G. Johnson, S. Fan, P. R. Villeneuve, J. D. Joannopoulos, and L. A. Kolodziejski, Phys. Rev. B 60, 5751 (1999).

${ }^{11}$ S. G. Johnson, P. R. Villeneuve, S. Fan, and J. D. Joannopoulos, Phys. Rev. B 62, 8212 (2000).

${ }^{12}$ J. Ctyroky, J. Opt. Soc. Am. A Opt. Image Sci. Vis. 18, 435 (2001).

${ }^{13}$ N. Y. Kim, P. Recher, W. D. Oliver, Y. Yamamoto, J. Kong, and H. Dai, Phys. Rev. Lett. 99, 036802 (2007).

${ }^{14}$ K. Iyakutti, A. Bodapati, X. Peng, P. Keblinsi, and S. K. Nayak, Phys. Rev. B 73, 035413 (2006).

${ }^{15}$ M. Kafesaki, M. M. Sigalas, and N. Garcia, Phys. Rev. Lett. 85, 4044 (2000).

${ }^{16}$ L. Martinelli, H. Benisty, O. Khayam, G. H. Duan, H. Heidrich, and K. Janiak, J. Lightwave Technol. 25, 2385 (2007).

${ }^{17}$ E. Viasnoff-Schwoob, C. Weisbuch, H. Benisty, C. Cuisin, E. Derouin, O. Drisse, G.-H. Duan, L. Legouézigou, O. Legouézigou, F. Pommereau, S. Golka, H. Heidrich, H. J. Hensel, and K. Janiak, Appl. Phys. Lett. 86, 101107 (2005).

${ }^{18}$ S. Olivier, H. Benisty, C. Weisbuch, C. J. M. Smith, T. F. Krauss, and R. Houdré, Opt. Express 11, 1490 (2003).

${ }^{19}$ S. Olivier, M. Rattier, H. Benisty, C. Weisbuch, C. J. M. Smith, R. M. De La Rue, T. F. Krauss, U. Oesterle, and R. Houdre,
Phys. Rev. B 63, 113311 (2001).

${ }^{20}$ O. Khayam, C. Cambournac, H. Benisty, M. Ayre, H. Brenot, G. H. Duan, and W. Pernice, Appl. Phys. Lett. 91, 041111 (2007).

${ }^{21}$ H. Kurt, H. Benisty, T. Melo, O. Khayam, and C. Cambournac, J. Opt. Soc. Am. B 25, C1 (2008).

${ }^{22}$ O. Khayam, H. Benisty, and C. Cambournac, Phys. Rev. B 78, 153107 (2008).

${ }^{23}$ S. Hughes, Opt. Lett. 29, 2659 (2004).

${ }^{24}$ R. A. Sepkhanov and C. W. J. Beenakker, Opt. Commun. 281, 5267 (2008).

${ }^{25}$ J. L. Garcia-Pomar, A. Cortijol, and M. Nieto-Vesperinas, Phys. Rev. Lett. 100, 236801 (2008).

${ }^{26}$ R. A. Sepkhanov, J. Nilsson, and C. W. J. Beenakker, Phys. Rev. B 78, 045122 (2008).

${ }^{27}$ A. Rycerz, J. Tworzydlo, and C. W. J. Beenakker, Nat. Phys. 3, 172 (2007).

${ }^{28}$ R. A. Sepkhanov, Y. B. Bazaliy, and C. W. J. Beenakker, Phys. Rev. A 75, 063813 (2007).

${ }^{29}$ F. D. M. Haldane and S. Raghu, Phys. Rev. Lett. 100, 013904 (2008).

${ }^{30}$ S. Zhang, B. Hu, P. Sebbah, and A. Z. Genack, Phys. Rev. Lett. 99, 063902 (2007).

${ }^{31}$ J. Topolancik, B. Ilic, and F. Vollmer, Phys. Rev. Lett. 99, 253901 (2007).

${ }^{32}$ I. Vorobeichik, E. Narevicius, G. Rosenblum, M. Orenstein, and N. Moiseyev, Phys. Rev. Lett. 90, 176806 (2003).

${ }^{33}$ E. Peral, A. Yariv, and L. Fellow, J. Lightwave Technol. 17, 942 (1999).

${ }^{34}$ A. Isacsson, L. M. Jonsson, J. M. Kinaret, and M. Jonso, Phys. Rev. B 77, 035423 (2008).

${ }^{35}$ H. Benisty, J. Appl. Phys. 79, 7483 (1996).

${ }^{36}$ E. Viasnoff-Schwoob, C. Weisbuch, H. Benisty, S. Olivier, S. Varoutsis, I. Robert-Philip, R. Houdré, and C. J. M. Smith, Phys. Rev. Lett. 95, 183901 (2005).

${ }^{37}$ J. B. Pendry, Low Energy Electron Diffraction (Academic, London, 1974).

${ }^{38}$ J. B. Pendry, Surf. Sci. 299-300, 375 (1994).

${ }^{39}$ S. Albaladejo, J. J. Saenz, M. Lester, L. S. Froufe-Pérez, and A. Garcia-Martin, Appl. Phys. Lett. 91, 061107 (2007).

${ }^{40}$ P. S. J. Russell, T. A. Birks, and F. D. Lloyd-Lucas, in Confined Electrons and Photons: New Physics and Applications, edited by E. Burstein and C. Weisbuch, NATO Advanced Studies Institute, Series B: Physics (Plenum, New York, 1995), Vol. 340, pp. 585. 